\title{
Preoperative evaluation for intraductal spread of breast cancer through current imaging tests: their strengths and limitations
}

\author{
Izo Kimijima
}

Published online: 3 October 2012

(C) The Japanese Breast Cancer Society 2012

With the growing use of mammography for routine breast cancer screening, tumors are now detected earlier and more frequently than ever before. Consequently, it is becoming increasingly important to define the exact location of early lesions to allow effective treatment through precision surgery.

The most common form of noninvasive breast cancerductal carcinoma in situ (DCIS) — is not generally noticed as a palpable tumor but as calcification during breast screening with mammography. DCIS generally does not metastasize, and thus, localized surgery and radiotherapy can be used to treat this lesion, thereby sparing most of the unaffected breast tissue. More invasive lesions, however, such as invasive ductal carcinoma (IDC), also require complete resection, including a margin of unaffected tissue, to ensure complete removal.

The situation is complicated by the coexistence of an intraductal component with IDC. If this intraductal lesion is missed during imaging when IDC is present, it may not be removed by subsequent surgery and can thus give rise to a positive surgical margin, increasing the likelihood of recurrence.

In this special feature, authors present a review of each imaging modality together with results of their own recent studies aimed at overcoming the limitations associated with the diagnosis of intraductal component coexisting with IDC and DCIS. Among the imaging tests considered is mammography, a routine screening methodology for breast cancer, which can detect DCIS primarily by identifying microcalcification. This calcification is also useful for

\footnotetext{
I. Kimijima $(\bowtie)$

Northern Fukushima Medical Center, Breast Center,

Fukushima, Japan

e-mail: kimijima0505@live.jp
}

confirming the complete resection of lesions by specimen radiography, especially in breast-conserving surgery. However, only half of DCIS lesions are associated with calcification, and even if the lesion does contain calcification, the gland can become deformed by compression during the procedure, making it difficult to identify the exact site of calcification in surgery.

Ultrasound examination is another commonly used imaging modality. Although it is considered to have poor reproducibility, it has high sensitivity and resolution for minute lesions, and the location of the lesion is easily projected onto the overlying skin. However, some DCIS lesions, without dilation of the duct, are missed by this diagnostic modality. In this edition, Nakajima et al. present results using B-mode, Doppler, and elastography for predicting intraductal components.

Magnetic resonance imaging (MRI) is now considered the most sensitive method for detecting DCIS lesions. Its ability to image without radiation exposure is a great advantage over computed tomography (CT). MRI is thus more frequently used to identify early lesions in case of suspicious findings during physical examinations or other diagnostic imaging examinations. Tozaki et al. review the use of MRI for the detection of DCIS lesions, including the new Breast Imaging-Reporting and Data System (BI-RADS) terminology for MRI.

Of particular note is the patient's prone position during the scan, which limits application of MRI in surgery. Thus, if the lesion has a feature that could be detected by sonography, its combination with MRI could be a significant advantage in identifying the precise location and area of the lesion.

Computed tomography is another effective imaging test for breast cancer. It is mainly used as a modality for staging of breast cancer, and it is not considered as the best 
imaging modality for DCIS lesions. In recent reports, it has been shown that these lesions could be detected by multidetector row computed tomography (MDCT). A major advantage of MDCT is that the patient can be tested in supine position, similar to that during surgery. Thus, the images obtained could be more applicable for guiding breast-conserving surgery (BCS) than MRI. Furthermore, with high-speed workstations and special imaging software, it may be possible to distinguish DCIS lesions from the surrounding normal breast tissue. This could contribute to successful complete resection of the lesion by BCS with minimal healthy tissue removal. Akashi-Tanaka discusses the merits of MDCT as applied to breast-conserving surgery in invasive and noninvasive lesions. These results have also been confirmed in DCIS, especially in high-grade lesions (Kimijima et al.).

Whilst existing imaging modalities can detect highgrade DCIS lesions relatively easily, precise detection of low-grade lesions is more difficult, as they appear similar to a normal gland. Anatomical knowledge of intraductal spreading could be very useful for obtaining a precise diagnosis in these cases.

Otake et al. present 3-dimensionally reconstructed pathological images of intraductally spreading lesions, and also show that ductal spreading into the ductal tree can be classified into 3 different types. In the most common type of ductal spreading, the intraductal component spreads to a single ductal tree to the nipple from an invasive tumor. Ductal trees are not always independent of each other and often have anastomoses between them, potentially allowing intraductal spreading. This new finding could provide a possible explanation for the extremely wide spreading of intraductal lesions. These authors review the pathological features of intraductal lesions, including their own data.

The authors hope that this special feature will contribute to the improvement of the diagnosis of DCIS, leading to more successful surgical outcomes. 